\title{
Erratum to: Surgical considerations with the operative fixation of unstable paediatric pelvic ring injuries
}

\author{
Mohamed Kenawey ${ }^{1}$
}

Published online: 8 May 2017

(C) SICOT aisbl 2017

Erratum to: International Orthopaedics (SICOT)

DOI 10.1007/s00264-017-3475-5

The presentation of Tables 1 and 3 in the original publication contain mistakes. The last row of the tables under Total of all columns are shifted one cell to the left. Below the corrected presentation:

Another correction, is the deletion of the duplicate reference number [23].

The original article was corrected.

Table 1 Modes of anterior and posterior pelvic ring failure

\begin{tabular}{|c|c|c|c|c|c|c|c|c|c|}
\hline & \multicolumn{9}{|l|}{ Posterior ring failure } \\
\hline & & $\begin{array}{l}\text { Trans- } \\
\text { iliac }\end{array}$ & $\begin{array}{l}\text { Trans- } \\
\text { sacral }\end{array}$ & $\begin{array}{l}\text { Trans-iliac }+ \text { contralat } \\
\text { trans-sacral }\end{array}$ & $\mathrm{SIJ}^{1}$ & $\begin{array}{l}\text { Trans-iliac fr/ } \\
\text { dislSIJI }^{2}\end{array}$ & $\begin{array}{l}\text { Trans-iliac fr/disl SIJ + } \\
\text { contralat SIJ }\end{array}$ & $\begin{array}{l}\text { Trans-sacral } \\
\text { fr/disl SIJ }\end{array}$ & Total \\
\hline \multirow{6}{*}{$\begin{array}{l}\text { Anterior ring } \\
\text { failure }\end{array}$} & Trans-pubic & 2 & 4 & 1 & 4 & 3 & 3 & 1 & 18 \\
\hline & Pubic symphysis & & 1 & & 3 & & & & 4 \\
\hline & $\begin{array}{l}\text { Trans-pubic }+ \text { pubic } \\
\text { symphysis }\end{array}$ & & & & 4 & & 1 & & 5 \\
\hline & Trans-acetabular & & & & $1^{*}$ & & & & 1 \\
\hline & $\begin{array}{l}\text { Trans-acetabular }+ \\
\text { pubic symphysis }\end{array}$ & & & & 1 & & & & 1 \\
\hline & Total & 2 & 5 & 1 & 13 & 3 & 4 & 1 & 29 \\
\hline
\end{tabular}

The online version of the original article can be found at http://dx.doi.org/ 10.1007/s00264-017-3475-5

Mohamed Kenawey

mohamed_kenawey@med.sohag.edu.eg

1 Orthopaedic Department, Sohag University Faculty of Medicine,

Sohag 82524, Egypt 
Table 3 Fixation devices for the anterior and posterior pelvic rings

Posterior ring failure

\begin{tabular}{|c|c|c|c|c|c|c|c|c|c|c|}
\hline & & None & $\begin{array}{l}\text { IS } \\
\text { screw }\end{array}$ & $\begin{array}{l}\text { Iliac } \\
\text { screw }^{*}\end{array}$ & $\begin{array}{l}\text { Ilio-iliac } \\
\text { bridge } \\
\text { platting }\end{array}$ & $\begin{array}{l}\text { Iliac } \\
\text { platting }\end{array}$ & $\begin{array}{l}\text { IS screws + } \\
\text { Iliac platting }\end{array}$ & $\begin{array}{l}\text { Iliac screws + } \\
\text { IS screws }\end{array}$ & $\begin{array}{l}\text { Lumbo-pelvic + ilio- } \\
\text { iliac bridge plating }\end{array}$ & Total \\
\hline \multirow{6}{*}{$\begin{array}{l}\text { Anterior } \\
\text { ring } \\
\text { failure }\end{array}$} & None & & 1 & & & 1 & 1 & & & 3 \\
\hline & $\begin{array}{l}\text { Supra-acetabular } \\
\text { external fixator }\end{array}$ & 8 & 6 & 3 & & 1 & & 2 & 1 & 21 \\
\hline & Symphyseal plating & & & & 1 & & & & & 1 \\
\hline & Pelvic brim plating & 1 & 2 & & & & & & & 3 \\
\hline & $\begin{array}{l}\text { Precut. acetabular } \\
\text { fixation }\end{array}$ & & 1 & & & & & & & 1 \\
\hline & Total & 9 & 10 & 3 & 1 & 2 & 1 & 2 & 1 & 29 \\
\hline
\end{tabular}

